\title{
Prognostic and predictive molecular biological markers in prostate cancer - significance of expression of genes PCA3 and TMPRSS2
}

\author{
R. SOUMAROVA ${ }^{1,2,5, *}$, A. BODAY ${ }^{3,6}$, V. KRHUTOVA ${ }^{3,6}$, A. JANOTOVA ${ }^{3,6}$, M. DVORAKOVA ${ }^{3,6}$, E. JALUVKOVA ${ }^{3,6}$, M. STURSA $^{4}$, H. PERKOVA ${ }^{1,5}$ \\ ${ }^{1}$ Comprehensive Cancer Centre, Department of Radiotherapy and Oncology, Novy Jicin, Czech Republic; ${ }^{2}$ Faculty of Medicine, University of \\ Ostrava, Department of Clinical Studies, Czech Republic; ${ }^{3}$ AGEL Laboratory, Department of Molecular Biology, genetics laboratory; ${ }^{4}$ Novy Jicin \\ Hospital and Comprehensive Cancer Centre, Department of Urology, Novy Jicin, Czech Republic; ${ }^{5}$ AGEL Research and Training Institute - Novy \\ Jicin Branch, Novy Jicin Hospital, Oncology Department; ${ }^{6}$ A GEL Research and Training Institute - Novy Jicin Branch, AGEL Laboratories, Czech \\ Republic
}

${ }^{\star}$ Correspondence: renata.soumarova@onkologickecentrum.cz

Received February 11, 2014 / Accepted April 14, 2014

\begin{abstract}
Prostate specific antigen and digital rectal examination have low specificity for detecting prostate cancer and they poorly predict the presence of aggressive disease. We present recent findings on PCA3 and TMPRSS:ERG fusion and assessed the relationship between PSA, urine PCA3 and TMPRSS2:ERG and corelation with pathological findings. We tested the PCA3 score in two groups. The first comprised 96 men treated in urology out-patient units with suspicion of prostate cancer, who had elevated PSA and/or positive DRE. The second group comprised 28 patients, who were treated by radiation for localised prostate cancer, and whose PCA3 was regularly monitored. A further cohort comprised patients with already-diagnosed tumors, who had undergone radical prostatectomy. With these, using histopathological samples, we examined samples of the TMPRSS2:ERG fusion gene and compared the results with Gleason score values and level of PSA. We also examined the TMPRSS2:ERG gene in patients who had positive biopsy. Part of the genetical analysis was also an examination of the MSMB gene.

The sensitivity of PCA3 testing was $66.7 \%$ and the specificity $78.5 \%$. TMPRSS2:ERG gene was correllated with the Gleason score. Neither the TMPRSS2:ERG $(\mathrm{p}=0.13)$ nor the MSMB $(\mathrm{p}=0.556)$ genotype had an influence on the value of the Gleason score. However a difference was found between the homozygote and wild type (WT) in the TMPRSS2 gene.

FISH analysis of TMPRSS/ERG gene fusion was evaluated as positive in $8(36.8 \%)$ of the biopsically verified tumors and in $20(37.3 \%)$ of the evaluated patients after RAPE of parafin slicing. We did not confirm a corellation between fusion and Gleason score $(\mathrm{p}=0.29)$.

$P C A 3$, with its higher sensitivity in comparison with PSA, is more useful for eventual screening examination. Identification of further molecular markers such as TMPRSS2, may be very promising ways to determine further prognosis of patients with prostate cancer.
\end{abstract}

Key words: prostate cancer, PSA, PCA3

Prostate cancer is the third most common tumor in men and its incidence continues to increase. In the past 20 years the number of recorded cases has more than trebled. The frequency of the occurrence of prostate tumors increases with age and in particular over the age of 60 , but the incidence of this disease among younger men is also on the increase. Often in the lower age groups more aggressive tumor behaviours are recorded. Most patients, however, have asymptomatic tumors and can live for a long time without signs of illness and die from other causes [1]. Mortality from prostate cancer has in fact remained approximately the same over the last 20 years [2].

The discovery of the prostate specific antigen (PSA), described by Wang in 1979, had a fundamental influence on the diagnosis, treatment and monitoring of prostate cancer.

PSA is a human kallikrein with an active neutral serine protease, which expresses in secreting epithelial cells, whose survival is dependent on androgens. The physiological function of PSA is the liquefaction of sperm, where its concentration is in the region of $0.2-0.5 \mathrm{mg} / \mathrm{ml}$. It gets into 
the bloodstream by passing across the barrier between the prostatic lumen and the capilliary blood. It exists in serum in two forms: free and bound with alfa 1 antichymotrypsin or alfa2 macroglobulin. PSA is at present the most clinically significant tumor marker for prostate cancer, however its higher count value in serum is, in addition to prostate cancer, also an indicator of inflammatory processes, mechanical irritation by instrumental examination in the area of the prostate etc.

The problem is above all its low specificity at values of 4-10 $\mathrm{ng} / \mathrm{ml}$, in the so-called diagnostical 'grey zone', in which prostate cancer appears in about $25 \%$ of patients [3]. PSA values leave a marked number of false-positive results, which in turn lead to a number of irrelevant biopsies. PSA testing is also one of the sources of the rise in the incidences of prostate cancer, in so far as it leads to the detection of even the asymptomatic forms. Full-body screening therefore remains controversial and is not unanimously recommended [4]. With the aim of increasing the sensitivity, specificity and predictive value of PSA, a range of other methods are used: PSA density (PSAD - ratio PSA/prostate volume), PSA velocity (PSAV- increase in serum concentrations of PSA over time), age-specific PSA (serum concentrations of PSA rise in relation to rising age) and especially the ratio of free/total PSA (f/t PSA).

The use of the above methods did indeed increase the specificity, but ideal results were not achieved, and the search continues. Attention is now concentrated at the microscopic level. In 1999 it was found that DD3 (PCA3) - differential display code 3 (prostate cancer antigen 3 ) is highly expressed in prostate cancer and not expresed in the normal prostate and in benign hyperplasia [5]. The product of this gene therefore has organ specificity and is closely connected with the occurrence of prostate cancer. The gene is localised on chromosome 9 in the area 9q21-22, and has 4 exons. The expression of PCA3 mRNA (messenger ribonucleic acid) was studied initially at cell level, in urine sediment and later in urine. The findings

show that its expression is limited only on the prostate tissue. With prostate cancer quantified values of $P C A 3 \mathrm{mRNA}$ are 66 times higher (middle value „upregulation“). During observation of PCA3 mRNA in urine sediment and in urine, the results of the examinations are influenced by the presence of normal cells and hence the so-called „PCA3“ score. This score expresses the ratio between PCA3 mRNA and PSA mRNA multiplied by a thousand. The combination of the results of the PCA3 test with those from the examination of further independent molecular markers (e.g. TMPRSS2:ERG fusion, SPINK1, GOLPH2) may increase the accuracy of a risk-assessment of the concret patient [6].

In recent years many studies have focussed on the relationship between the presence of fusion gene TMPRSS2:ERG in prostate tumors and the risk of the emergence of progression of this tumor. The TMPRSS2 gene encodes transmembrane serin protease, which is continuously expressed in the prostate, under androgen transscription control. The incidence of this fusion is relatively frequent. Current studies show the capture of TMPRSS2:ERG fusion using the FISH method in approximately $40 \%$ of prostate tumor cases $[7,8]$. The FISH method enables the identification of the mechanism of the emergence of this rearrangement and also the number of copies of the fusion gene.

A common genetic variant (rs10993994) in the 5 ' region of the gene encoding $\beta$-microseminoprotein (MSMB) is associated with circulating levels of MSMB and prostate cancer risk [9]. Whether MSMB levels are predictive of prostate cancer risk has been evaluated.

All these factors, as evident from already published studies, should play a definite role in the progression of the illness, its recurrence and death caused by prostate cancer $[10,11]$. Prostate cancer is a relatively heterogeneous illness. At present we are not able to distinguish a man in whom the tumor will develop in an aggressively metastatic way from a man in whom this will not happen. There are however indications that it is indeed the presence of the TMPRSS2:ERG gene which may be a predictor of a more agressive form of illness and death as a result of prostate cancer. On the basis of this each patient could receive an individually-designed appropriate treatment. The question is then if, in patients with higher PSA counts and/or abnormal rectal examination (DRE) who would be candidates for primary biopsy, this invasive method can be substituted by PCA3 testing. And in addition if, in patients with elevated PSA and negative biopsies, it is important to repeat these biopsies. Moreover, if, in patients with hitherto positive biopsies, it is possible, in combination with other results, to distinguish an indolent from an aggressive tumor.

The purpose of our study was to test the sensitivity of $P C A 3$ and efforts to find out whether you can refine indications for repeated biopsy in patient with elevated PSA. Correlations of $P C A 3$ and gene TMPRSS2:ERG with histopathological findings then help ídentify the prognostic adverse patient groups. The development of the disease in tested patients will continue to be monitored.

\section{Material and methods}

We tested the PCA3 score in two groups. The first group comprised 96 men treated in urology out-patient units with suspicion of prostate cancer, who had elevated PSA and/or positive DRE. The second group comprised 28 patients, who were treated by radiation for localised prostate cancer, and whose $P C A 3$ was regularly monitored. The patients gave blood and urine samples. In the first group we compared PCA3 with the biopsy result and established its specificity and sensitivity. In the group of treated patients we tested the predictive value of $P C A 3$, which we tested before, during and after each regular check-up every 3 months after termination of treatment.

A further cohort comprised patients with already-diagnosed tumors, who had undergone radical prostatectomy. With these, using histopathological samples, we examined samples of the TMPRSS2:ERG fusion gene and compared the results with Gleason score values and level of PSA. We also examined the TMPRSS2:ERG gene in patients who had positive 


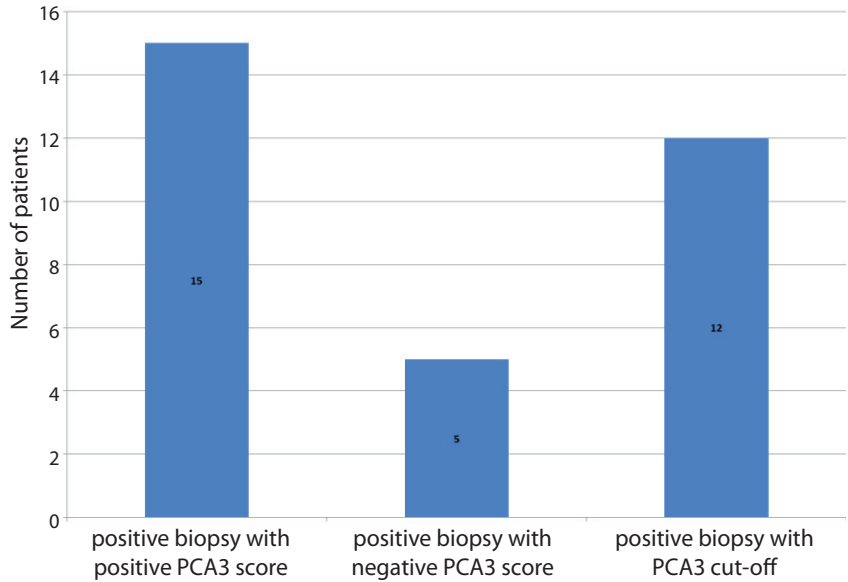

Figure 1. Number of patients with biopsy and PCA3 score This graph represents the number of patients with positive biopsy and PCA scores (positive, negative and cut-off = grey zone)

biopsy. Part of the genetical analysis was also an examination of the MSMB gene, which encodes $\beta$-seminomicroprotein. DNA was isolated from uncoagulated blood in a Biorobot M48 (Qiagen) automatic isolator using MagAttract DNA Blood Mini M48 kit (Qiagen).

Polymorphisms rs10993994 in the MSMB gene and rs12329760 (Met160Val SNP) in the TMPRSS gene were detected using the real-time PCR (LC480Roche) - SNP-assay method.

Analysis of $P C A 3$ was conducted with a closed Progensa $P C A 3$ system according to protocol. The protocol precisely describes the correct collection, including prostate massage and urine preservation and individual steps in the analysis. An individual analysis starts with the capture of PCA RNA using an oligomer bound to magnetic particles. This step is followed by specific PCR „Transcription-mediated amplification", and then hybridisation and after this signal detection. The examination results are given as a "PCA score" which is determined based on the ratio of PCA3 RNA to PSA RNA multiplied by 1000 . The presence of TMPRSS-ERG fusion was monitored using FISH on histological preparations using a fluorescently-marked probe (Poseidon TMPRSS2:ERG probe, Kreatech Diagnostics, The Netherlands). For the statistical data analysis SPSS version 15 software was used (SPSS Inc., Chicago, USA). In categorical parameters the subsets were compared using a Chi-squared test: in this case Fisher's exact test. To compare the quantitive parameters, according to the normality of the data, a paired Student t-test was used, in particular the Mann-Whitney U-test. The normality of the data was confirmed using a Shapiro-Wilk test.

\section{Results}

In the patient group with suspected prostate cancer, the tumor was biopsically confirmed in 32 men (30.7\%). In the whole

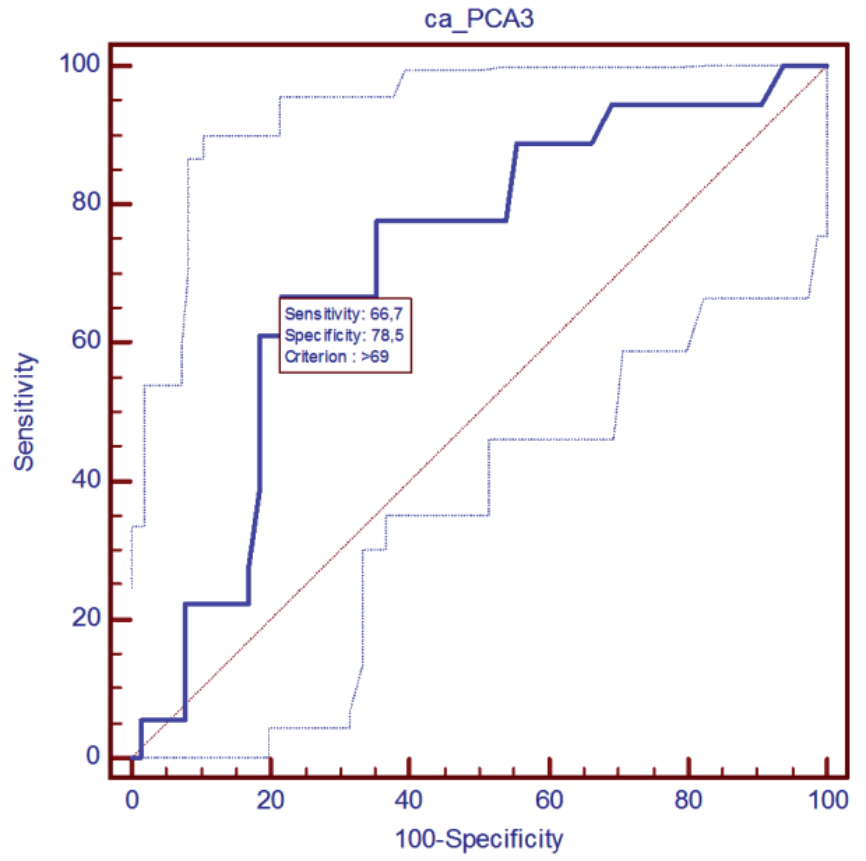

Figure 2. Sensitivity and specificity of $P C A 3$

Receiver operating characteristic (ROC) curves of the prostate cancer antigen 3 (PCA3) gene in the whole group $(n=96)$

group 96 men had their PCA3 score taken: in 19 men (19.8\%) it was evaluated as negative, in 28 cases (29.2\%) PCA-score values fluctuated within the grey zone. Other patients (49) were positive (51\%). The distribution of patients with positive biopsies based on PCA3 counts is given in Figure 1.

We established the sensitivity and specificity of $P C A 3$ using ROC (Receiver Operating Characteristic) curves in the patient group with and without diagnosed prostate cancer

(Figure 2). The sensitivity was $66.7 \%$ and the specificity $78.5 \%$.

In the patient group which underwent radiation therapy for localized prostate cancer, very little cellularity was once again found in the urine, and the PCA 3 score was thus unmeasurable. In patients with prostate cancer after radical prostatectomy or with positive biopsy we examined the TMPRSS2:ERG gene and correllated it with the Gleason score (GS) using a KruskalWallis test. Neither the TMPRSS2:ERG $(\mathrm{p}=0.13)$ nor the MSMB $(\mathrm{p}=0.556)$ genotype had an influence on the value of the Gleason score. However a difference was found between the homozygote and wild type (WT) in the TMPRSS2 gene (Figure 3). Positive TMPRSS2:ERG fusion was found in $36.4 \%$ of patients of percutaneous biopsy and $37.3 \%$ of patients of parafin section.

Using the Mann-Whitney test the relationship was statistically significant between GS and WT versus homozygote TMPRSS2:ERG. In WT the median Gleason score was statistically significantly higher $(\mathrm{p}=0.0489)$.

FISH analysis of TMPRSS:ERG gene fusion was evaluated as positive in $8(36.8 \%)$ of the biopsically verified tumors 
and in $20(37.3 \%)$ of the evaluated patients after RAPE of parafin slicing. We did not confirm a corellation between fusion TMPRSS:ERG and MSMB and Gleason score ( $\mathrm{p}=0.29$, $\mathrm{p}=0.14)$.

\section{Discussion}

Examination of PSA in serum still belongs among the fundamental biomarkers for detection and monitoring of prostate cancer. It however has a lower specificity for detection of prostate cancer and is a very poor predictor of aggressive tumors. Examination on urine is non-invasive and is an anticipated source of new biomarkers which may be more sensitive and specific [11]. Among the anticipated markers are DPD 3 and TMPRSS2:ERG gene fusion [6]. In our group we found $67 \%$ sensitivity and $79 \%$ specificity in PCA3. PCA3 is significantly higher in patients with prostate cancer than with benign hyperplasia [13].

On the basis of ROC analyses it is possible to conclude a usefulness of this non-invasive test as a predictor of biopsy results in wider clinical practice.

On evalutation of the relationship between PCA3 and tumor agressiveness (GS) it was possible to show a dependency, similar to other studies [14]. However some data do show a dependency between $P C A 3$ score and extracapsular spread and tumor volume [15].PCA3 is probably not useful either for monitoring success of therapy, as in our patients there was very low cellularity in urine. With treatment the cellularity of urine decreases, as does the level of the RNA genes PCA3.

The fusion gene TMPRSS2:ERG appears in patients with prostate cancer, whereas in the group with hyperplasia it is not detected. In an American study the gene was present in 46\% of men with a positive biopsy, in $0 \%$ of men with a negative finding $[16,17]$. In our group we observed positivity of the fusion gene in $36.4 \%$ of biopsies and in $37.3 \%$ of patients from histological post-operational samples, which corresponds to published findings. The positivity may mean a higher stage of illness [18], which we were not able to demonstrate in relation to the patient count in the different risk groups. The presence of the wild type of gene TMPRSS2 may be a prognostic mark of higher tumor aggressiveness. In our group these patients had higher GS.

In the future in our patient group we want to evaluate survival related to positive TMPRSS2:ERG fusion. Retrospective analysis of Canadian authors shows that TMPRSS2:ERG fusion and simultaneous PTEN loss may be a predictor of early biochemical recurrence $[19,20]$. The combination of the individual genotypes under study however does not increase the predicitve value of diagnosis in specific patients. This is due to the heterogeneity of the tumor caused by many influences of different genes with defective expressions and ultimately by the genetic background of the body.

The presence of fusion may help to confirm the presence of prostate cancer from biopsy, however it does not determine its prognosis. A combination of PCA3 and TMPRSS2

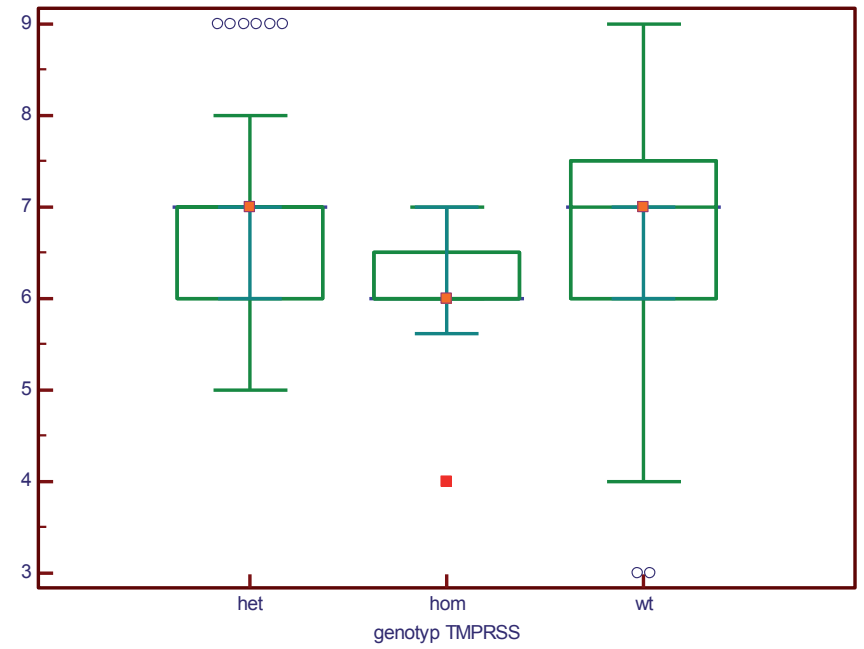

Figure 3. Dependency of Gleason score on TMPRSS genotype This graph demonstrates that the genotype TMPRSS does not affect the value of the Gleason score. On the other hand there is significant difference between wild type and homozygot.

wt - wild type, hom - homozygot, het - heterozygot

with PSA and DRE can help to identify patients indicated for biopsy [21].

\section{Conclusion}

$P C A 3$, with its higher sensitivity in comparison with PSA, is more useful for eventual screening examination, but a disadvantage may be its price. However repeated biopsies with negative results may be more stressful for the patient and ultimately more expensive. $P C A 3$, as a part of the pre-biopsy nomogram, maybe useful to indicate biopsy.

Identification of further molecular markers such as TMPRSS2, PTEN, MSMB etc. may be very promising ways to determine further prognosis of patients with prostate cancer.

\section{Recomendation}

Among candidates for $P C A 3$ examination should be the following:

- Men with elevated PSA with no evidence of cancer at the first biopsy

- Men with elevated PSA with different degrees of prostatitis

- Men who are actively monitored for microfocal cancer

\section{References}

[1] FREEDLAND SJ. Screening, risk assessment, and the approach to therapy in patients with prostate cancer. Cancer. 2011; 117: 1123-1135. http://dx.doi.org/10.1002/cncr.25477 
[2] www.svod.cz

[3] SABRA, R. et al.: Prostate cancer until 2000. MaxdorfJessenius, Prague 1996, 63 s.

[4] MOYER VA. Screening for Prostate Cancer: U.S. Preventive Services Task Force Recommendation Statement. Ann Intern Med. 2012; 157: 120-134. http://dx.doi.org/10.7326/00034819-157-2-201207170-00459

[5] BUSSMAKERS MJ, VAN BOKHOVEN A, VERHAEGH GW, SMIT FP, KARTHAUS HF, et al. DD3: a new prostate specific gene, highly overexpressed in prostate cancer. Cancer Res. 1999; 59: 5975-5979.

[6] SALAGIERSKI M, SCHALKEN JA. Molecular diagnosis of prostate cancer: PCA3 and TMPRSS2: ERG gene fusion. J Urol. 2012; 187: 795-801. http://dx.doi.org/10.1016/j. juro.2011.10.133

[7] ROOBOL MJ, HAESE A, BJARTELL A. Tumor markers in prostate cancer III: biomarkers in urine. Acta Oncol. 2011; 50 Suppl 1: 85-89. http://dx.doi.org/10.3109/0284186X.2010.52 $\underline{4935}$

[8] HOSSAIN D, BOSTWICK DG. Significance of the TMPRSS2: ERG gene fusion in prostate cancer. BJU Int. 2013; 111: 834-835. http://dx.doi.org/10.1111/bju. 12120

[9] HAIMAN CA, STRAM DO, VICKERS AJ, WILKENS LR, BRAUN K, et al. Levels of beta-microseminoprotein in blood and risk of prostate cancer in multiplepopulations. J Natl Cancer Inst. 2013; 105: 237-243. http://dx.doi.org/10.1093/jnci/djs486

[10] SQUIRE JA. TMPRSS2-ERG and PTEN loss in prostate cancer. Nat Genet. 2009; 41: 509-510. http://dx.doi.org/10.1038/ ng0509-509

[11] CARVER BS, TRAN J, GOPALAN A, CHEN Z, SHAIKH S, et al. Aberrant ERG expression cooperates with loss of PTEN to promote cancer progression in the prostate. Nat Genet. 2009; 41: 619-624. http://dx.doi.org/10.1038/ng.370

[12] TRUONG M, YANG B, JARRARD DF. Toward the detection of prostate cancer in urine: a critical analysis. J Urol. 2013; 189: 422-429. http://dx.doi.org/10.1016/j.juro.2012.04.143

[13] KLECKA J, HOLUBEC L, PESTA M, TOPOLCAN O, HORA $\mathrm{M}$, et al. Differential display code 3 (DD3/PCA3) in prostate cancer diagnosis. Anticancer Res. 2010; 30: 665-670.
[14] AUGUSTIN H, MAYRHOFER K, PUMMER K, MANNWEILER S. Relationship between prostate cancer gene 3 (PCA3) and characteristics of tumor aggressiveness. Prostate. 2013; 73: 203-210. http://dx.doi.org/10.1002/ pros. 22558

[15] WHITMAN EJ, GROSKOPF J, ALI A, CHEN Y, BLASE A, et al. PCA3 score before radical prostatectomy predicts extracapsular extension and tumor volume. J Urol. 2008; 180: 1975-1978; discussion 1978-9.

[16] MOSQUERA JM, MEHRA R, REGAN MM, PERNER S, GENEGA EM, et al. Prevalence of TMPRSS2-ERG fusion prostate cancer among men undergoing prostate biopsy in the United States. MG. Clin Cancer Res. 2009; 15: 4706-4711. http://dx.doi.org/10.1158/1078-0432.CCR-08-2927

[17] CORNU JN, CANCEL-TASSIN G, EGROT C, GAFFORY C, HAAB F, et al. Urine TMPRSS2: ERG fusion transcript integrated with PCA3 score, genotyping, and biological features are correlated to the results of prostatic biopsies in men at risk of prostate cancer. Prostate. 2013; 73: 242-249. http://dx.doi. org/10.1002/pros.22563

[18] TAVUKCU HH, MANGIR N, OZYUREK M, TURKERI L. Preliminary Results of Noninvasive Detection of TMPRSS2: ERG Gene Fusion in a Cohort of Patients With Localized Prostate Cancer. Korean J Urol. 2013; 54: 359-63. http://dx.doi. org/10.4111/kju.2013.54.6.359

[19] YOSHIMOTO M, JOSHUA AM, CUNHA IW, COUDRY RA, FONSECA FP, et al. Absence of TMPRSS2: ERG fusions and PTEN losses in prostate cancer is associated with a favorable outcome. Mod Pathol. 2008; 21: 1451-1460. http://dx.doi. org/10.1038/modpathol.2008.96

[20] NAMI RK, SUGAR L, WANG Z, YANG W, KITCHING R, et al. Expression of TMPRSS2: ERG gene fusion in prostate cancer cells is an important prognostic factor for cancer progression. Cancer Biol Ther 2007; 6: 40-45. http://dx.doi. org/10.4161/cbt.6.1.3489

[21] DIMITRIADIS E, KALOGEROPOULOS T, VELAETI S, SOTIRIOU S, VASSILIOU E, et al. Study of genetic and epigenetic alterations in urine samples as diagnostic markers for prostate cancer. Anticancer Res. 2013; 33: 191-197. 